\title{
环境化学与四大基础化学教学衔接的探究
}

\author{
汪群慧 $1,2,{ }^{*}$, 宋娜 2 , 高明 ${ }^{1}$, 吴川福 ${ }^{1}$, 梁宝瑞 ${ }^{2}$ \\ 1 北京科技大学能源与环境工程学院, 北京 100083 \\ 2 北京科技大学天津学院, 天津 301830
}

\begin{abstract}
摘要: 环境工程专业必修课程环境化学与该专业四大基础化学课程(无机化学、有机化学、物理化学、分析化学)在教学 内容上存在重复现象。为提高课堂教学效率和学生的学习积极性, 本教学团队总结分析了环境化学与四大基础化学的相 似知识点, 采用更新优化教学内容, 开展提问式、对比式、讨论式等多种教学方式, 引导学生由 “已知” 探 “新知”, 使知识层层深化, 并利用 “雨课堂” 的教学模式助力教学衔接, 提高教学效果。
\end{abstract}

关键词: 环境化学; 基础化学; 重复内容; 教学衔接; 教学改革

中图分类号: G64; O6

\section{Study on the Teaching Connection between Environmental Chemistry and Four Basic Chemistry Courses}

\author{
Qunhui Wang ${ }^{1,2,{ }^{*}, \text { Na Song }}{ }^{2}$, Ming Gao ${ }^{1}$, Chuanfu Wu ${ }^{1}$, Baorui Liang ${ }^{2}$ \\ ${ }^{1}$ School of Energy and Environmental Engineering, University of Science and Technology Beijing, Beijing 100083, China. \\ ${ }^{2}$ Tianjin College, University of Science and Technology Beijing, Tianjin 301830, China.
}

\begin{abstract}
As a required course for the environmental engineering major, environmental chemistry has overlapped teaching content with the four major basic chemistry courses (inorganic chemistry, organic chemistry, physical chemistry and analytical chemistry). In order to improve classroom teaching efficiency and students' learning enthusiasm, our teaching team summarizes and analyzes the similar knowledge between environmental chemistry and the four basic chemistry courses, and then updates and optimizes the teaching content. Meanwhile, with assorted teaching methods including questioning, comparing and discussion, we guide students to explore "new knowledge" from "known knowledge" to deepen knowledge layer by layer. Furthermore, the "rain classroom" teaching mode is applied to help the teaching connection, which can improve the teaching effect.
\end{abstract}

Key Words: Environmental chemistry; Basic chemistry; Repeated content; Teaching connection; Teaching reform

环境学科的三大支柱是化学、生物和力学 ${ }^{[1]}$, 故环境类专业一般都将四大基础化学(无机化学、 有机化学、物理化学、分析化学)和环境化学分别作为学科基础课和专业必修课开设。环境化学的教 学内容与四大基础化学之间不可避免地出现了一些相似或重复内容, 浪费较为紧张的授课时间, 影 响教学效率和效果。因此, 合理安排环境化学的教学内容, 有效实现其与四大基础化学的高效衔接, 巧妙处理好知识的阶段性、延续性和整体性, 激发学生的学习兴趣和对专业的热爱, 对环境化学教 学来说是一个严峻的挑战。本教学团队同时承担了北京科技大学能源与环境工程学院、北京科技大

收稿: 2020-02-21; 录用: 2020-04-06; 网络发表: 2020-04-13

*通讯作者, Email: wangqh59@sina.com

基金资助：2019 年北京高等教育 “本科教学改革创新项目” ; 北京科技大学天津学院第五批本科教育教学改革面上项目(JY201904) 
学天津学院环境工程专业的环境化学课程(32 学时理论课 +8 学时实验课), 主要选用的是汪群慧等 ${ }^{[2]}$ 主编的《环境化学》第 2 版(2008 年)教材, 同时参考选用戴树桂 ${ }^{[3]}$ 主编的《环境化学》第 2 版及其 他教材的部分内容。本文通过总结和归纳四大基础化学与环境化学的相似及重复内容, 探讨其产生 的原因和由此引起的不良影响, 研究教学内容的有效衔接方式, 提出相应的教学改革措施, 为环境 化学教学内容和模式改革提供有意义的参考。

\section{1 环境化学与四大基础化学之间的相似内容与分析}

环境化学主要研究有害化学物质在环境介质(大气、水体、土壤、生物)中的存在形态、化学特 性、行为效应及控制的化学原理和方法, 兼具环境与化学两大学科的知识点。环境化学是后续相关 专业课程的理论基础, 良好的环境化学知识储备能够为分析解决一般环境问题提供理论支持。北京 科技大学采用的四大基础化学和环境化学教材如表 1 所示, 其课程内容存在的相似或重复内容如表 2 所示。

表 1 北京科技大学采用的四大基础化学和环境化学教材

\begin{tabular}{|c|c|c|}
\hline 教材名称 & 编者 & 出版社 \\
\hline 无机化学(第 5 版 $)^{[4]}$ & 大连理工大学无机化学教研室 & 高等教育出版社, 2006.5 \\
\hline \multirow[t]{2}{*}{ 分析化学(第 6 版) ${ }^{[5]}$} & 华东理工大学分析化学教研组, 四川大学工 & 高等教育出版社, 2009.7 \\
\hline & 科化学基础课程教学基地 & \\
\hline 有机化学(第 4 版) ${ }^{[6]}$ & 汪小兰 & 高等教育出版社, 2005.5 \\
\hline 物理化学(第 5 版)上下册 ${ }^{[7]}$ & 傅献彩, 沈文霞, 姚天扬, 等 & 高等教育出版社, 2005.7 \\
\hline 环境化学(第 2 版) ${ }^{[2]}$ & 汪群慧, 王遂, 苏荣军, 等 & 哈尔滨工业大学出版社, 2008.1 \\
\hline
\end{tabular}

表 2 环境化学与四大基础化学教材中部分相似或重复的知识点

\begin{tabular}{|c|c|c|c|c|c|}
\hline \multirow{2}{*}{ 知识点 } & \multicolumn{5}{|c|}{ 部分知识点所在各教材中的章节 } \\
\hline & 环境化学 & 无机化学 & 分析化学 & 有机化学* & 物理化学 \\
\hline 键能 & 2.1 和 3.1 & 9.6 .2 & & & \\
\hline 酸碱平衡 & 5.2 & 5.3 & 4.1 & & \\
\hline 沉淀平衡 & 6.1 & 6 & 7.3 & & \\
\hline 配合作用 & 6.2 & 5.7 和 5.8 & 5.1 & & \\
\hline 氧化还原作用 & 6.3 & 7 & 6.1 & & 9 \\
\hline 物质形态分布曲线 & 5.2 & & 4.2 & & \\
\hline 亨利定律 & 5.1 & & & & 4.6 \\
\hline 界面过程 & 7.2 & & & & 11 \\
\hline 碳氢化合物的生物转化 & 2.3 & & & $2,3,4,8,9,10$ & \\
\hline 典型有机污染物 & 15 & & & 4,6 & \\
\hline
\end{tabular}

“环境化学 2.3 节 “碳氢化合物生物转化” 与有机化学较多章节有交叉及重复内容, 体现在反应机理方面; 环境化学第 15 章

“典型有机污染物” 与有机化学第 4 章 “环烃” 及第 6 章 “卤代烃” 虽有重复, 但环境化学更侧重于介绍有机污染物的来 源、迁移、危害及防治, 而有机化学侧重于每种有机污染物的性质、相关反应及反应机理

\section{2 教学内容相似或重复产生的不良影响}

环境化学授课学时为 32 学时, 授课时间少、任务重。为保证课程知识体系的完整性, 教师可能 会对重复内容再一次进行详细讲解, 而课程重点难点知识的授课学时反而被压缩, 造成授课学时的 
浪费; 将学生已经掌握的知识再次重复讲解, 很容易引起学生的厌烦心理, 打消学生学习积极性, 从而引发上课睡觉、看小说、玩手机等不良现象; 而消极的课堂氛围又会降低授课教师的授课热情, 影响课堂教学效率和效果。因此, 合理安排环境化学教学内容, 实现与四大基础化学的有效衔接, 巧妙处理好知识的阶段性、延续性和整体性, 激发学生的学习兴趣和对专业的热爱, 对环境化学的 教学来说具有重要意义。

\section{3 环境化学与四大基础化学有效衔接的措施}

\section{1 更新优化教学内容, 找好衔接切入点}

\subsection{1 精炼基础知识, 删减重复内容}

由于教学学时的压缩, 环境化学教材涵盖的所有内容通常无法在课堂上进行全部讲解, 应对一 些基础的化学理论进行适当精简, 避免重复。例如在水环境化学一章中, 污染物的溶解沉淀、氧化 还原、配位等重点反应, 由于在无机化学和分析化学中已重点讲解过, 所以在环境化学学习中会以 提问的方式引导学生回顾复习这些基础知识, 然后结合环境要素与各反应的联系引入新知识点, 重 点放在如何使学生把基础化学反应理论和化学思维应用在环境保护与环境治理中, 拓宽学生的知识 面。

\subsection{2 优化内容层次, 做好内外衔接}

鉴于环境化学与各基础化学知识存在多源融合和多层交叉关系, 以环境化学课程内容为主体, 对外应强化学科交叉关系分析和整理, 对内应优化内容层次和章节排布, 做到精准教学、有的放矢, 具体方法如下。一是总结分类交叉内容。按照章节内容与各基础化学的交叉关系和融合程度进行整 理分类, 明确内容对应关系。二是优化章节内容排序。在倡导相同交叉学科集中课时教学的基础上, 综合考虑章节重要度及学习难度, 合理调整各章节的设置顺序。如讲到天然水组成和性质时, 可以 将其中 $\mathrm{O}_{2}$ 和 $\mathrm{CO}_{2}$ 的溶解度计算归到水环境中污染物在大气-水间亨利系数去讲, 进而用分配系数的 概念归纳液(气)相与固相之间的吸附与脱附平衡、气相与液相之间的吸收与挥发平衡、水相与辛醇 相之间的萃取与反萃取平衡等, 从而使学生能较系统全面地掌握污染物的相间迁移行为和规律。

\subsection{3 拓展课堂内容, 提升学生趣味}

化学史和环境化学家的介绍对于提升环境化学的教学效果具有促进作用, 其不仅可以帮助学生 了解所学理论的提出过程, 同时也可以提升学科趣味性, 激发学生的学习兴趣 ${ }^{[8]}$ 。如在讲到臭氧层 破坏时, 先给学生放一段小视频—— “臭氧的前世今生” , 使学生了解大气臭氧层的主要特征, 然 后讲授平流层臭氧的生成与破坏机理, 中间穿插三位科学家(罗兰、莫利纳、克鲁陈)如何发现该机 理, 从而获得诺贝尔化学奖的故事, 提升课堂的趣味性, 增加学生的学习热情, 同时激励学生的科 研兴趣。

\section{2 多种教学方法并举, 促进教学衔接}

\subsection{1 开展提问式教学}

教师应明确环境化学与四大基础化学的衔接内容, 对于涉及基础化学相关知识的授课内容, 教 师可通过提出一系列综合性问题, 调动学生思维、促使学生主动将相关知识衔接起来。如在讲到硫 酸型烟雾或酸雨形成机理时, 首先提出物理化学中已讲过的物质结构相关问题: $\mathrm{SO}_{2}$ 和 $\mathrm{NO}$ 的空间 构型(杂化轨道)是怎样的? 从而解释前者溶于水后者不溶于水的道理, 进而延伸到 $\mathrm{SO}_{2} \cdot \mathrm{H}_{2} \mathrm{O}$ 在水中 以几种形态存在? (主要是三种: $\mathrm{SO}_{2} \cdot \mathrm{H}_{2} \mathrm{O} 、 \mathrm{HSO}_{3}^{-} 、 \mathrm{SO}_{3}^{2-}$ ), 接下来讲解附着在大气或雾滴中的 $\mathrm{S}(\mathrm{IV}$ ), 在 $\mathrm{O}_{2} 、 \mathrm{O}_{3} 、 \mathrm{H}_{2} \mathrm{O}_{2}$ 、各种自由基的氧化作用和雾滴中锰、铁及氨的催化作用下被加速氧化为 $\mathrm{S}(\mathrm{VI})$, 形成硫酸或硫酸盐, 再与大气中的颗粒物等形成硫酸型烟雾; 而 $\mathrm{NO}$ 首先需要气相氧化转化为 $\mathrm{NO}_{2}$ 后, 遇水生成硝酸和亚硝酸, 成为硫酸烟雾或酸雨的组分之一。再进一步拓展到烟气处理过程中常 采用液相中湿法脱硫和气相中催化脱硝的原理, 从而使学生的认知水平呈螺旋式上升。 


\subsection{2 实施对比式教学}

对于教学过程中不可避免的重复内容, 可采用对比教学法, 授课教师将不同课程间重复内容的 不同侧重点清晰有效地区分出来, 加深学生对知识点的理解。如四大基础化学侧重于介绍化学物质 的反应原理, 而环境化学则应侧重讲解环境污染物的迁移转化机理及其在环境学科领域中的应用。 授课教师采用 “对比、类比” 的方法减少知识认知梯度, 使学生的理解和记忆变得更加清晰、牢固, 达到事半功倍的效果。

如讲到污染物的水解反应时, 可将它与水中酸碱平衡的电离反应作对比, 说明盐类的水解, 就 是盐电离出的弱离子与水电离出来的 $\mathrm{H}^{+}$或 $\mathrm{OH}^{-}$反应, 从而促进了水的电离。进一步引申到金属离子 的水解反应与盐的水解反应实质是一样的, 其过程是水合金属离子 $\left(\left[\mathrm{Me}\left(\mathrm{H}_{2} \mathrm{O}\right)_{n}\right]^{2+}\right)$ 中的水分子离开金 属离子, 与水电离出来的 $\mathrm{H}^{+}$结合形成 $\mathrm{H}_{3} \mathrm{O}^{+}$, 而水合金属离子与水电离出来的 $\mathrm{OH}^{-}$结合形成 $\left[\mathrm{Me}\left(\mathrm{H}_{2} \mathrm{O}\right)_{n-1}(\mathrm{OH})\right]^{+}$。因此, 从反应形式上看, 也可认为是 $\mathrm{OH}^{-}$置换了金属离子水合分子的反应 $\left.\left[\mathrm{Me}\left(\mathrm{H}_{2} \mathrm{O}\right)_{n}\right]^{2+}+\mathrm{H}_{2} \mathrm{O} \leftrightharpoons\left[\mathrm{Me}\left(\mathrm{H}_{2} \mathrm{O}\right)_{n-1}(\mathrm{OH})\right]^{+}+\mathrm{H}_{3} \mathrm{O}^{+}\right)$。有机物的水解反应也是其电离的两部分, 分别 与水电离出来的 $\mathrm{H}^{+}$或 $\mathrm{OH}^{-}$反应, 其反应的实质, 也可认为是 $\mathrm{OH}^{-}$置换了有机物的官能团 $\mathrm{X}^{-}$的反应 $\left(\mathrm{R}-\mathrm{X}+\mathrm{H}_{2} \mathrm{O} \rightarrow \mathrm{R}-\mathrm{OH}+\mathrm{HX}\right)$ 。

类似例子很多, 只要授课教师重视和熟悉知识的衔接, 善于联系以前学生已有知识, 通过类比 引导, 引导学生由 “已知” 探 “新知”, 使知识循序渐进、层层深化。

\subsection{3 设置讨论式学生讲堂}

在教学过程中也可改变传统的以教师为中心的 “灌输式” 教学方式, 设置学生讲堂, 让学生自 己查找资料, 准备 PPT 上台讲解知识点, 充分发挥学生的主动性。教师可以出一些供学生讨论的专 题, 如光化学反应与无机化学和物理化学中学过的热化学反应在分子活化、电子分布及构型、产物 能量等方面有何不同? 大气气溶胶的性质与物理化学中学过的溶液胶体性质有何异同? 如何利用表 面化学的原理解释挥发性有机物(VOC)形成 PM 2.5 的机制? 四大化学中学过的氧化还原反应在污染 物处理方面的应用有哪些? 光化学烟雾和硫酸型烟雾的异同点有哪些? 在研究环境污染物迁移转化 规律时如何应用化学反应动力学等等。学生也可以自己选题, 对于一些重复知识点划分几个小组, 每组选定一个课题, 通过查阅文献, 经讨论、归纳、整理、分析和写作等多个环节, 做成 PPT 向大 家展示, 这样不仅保持了教学内容的连贯性, 又能促进学生自主复习, 加深印象。汇报采取台上学 生讲解、台下同学提问、授课教师点评的方式, 加强学生与教师之间的交流, 实现知识的有效碰撞 与传递, 培养学生运用已有知识解决实际问题的能力, 从而有效促进学生自主参与环境化学与四大 化学的教学衔接。

\section{3 采用 “雨课堂” 线上线下混合教学, 助力教学衔接}

“雨课堂” 是以手机为载体进行的一项线上线下一体化的教学设计, 为课前-课上-课后的每一 个教学环节都提供了完整立体的数据支持, 实质性解决 “三率” 即到课率、抬头率、入脑率问题。

课前, 对学生之前已有基础的、四大化学中讲到过的一些易理解的知识, 可以将教学内容做成 “预习教案” (包括慕课、PPT、相关视频等), 通过 “雨课堂” 的预习功能推送给学生, 教师可以通 过平台了解学生预习进展情况。如: 可将金属离子的水解反应、溶解沉淀反应、配位反应发给学生 预习, 让学生找到他们之间的相似点和关联性。

课堂上, 幻灯片同步到学生手机进行授课并随时测试。对于学生难理解的知识, 以启发式教学 法和练习法等多种方法相结合来进行知识的巩固和提高。可以总结归纳学生预习中的问题, 如: 可 设个两个投票题(即是非题): 金属离子的水解反应是否可看作水中的羟基配体取代水分子配体的反 应: (A) 是; (B) 否; 金属离子的沉淀反应是否看作是与金属离子形成溶度积较小沉淀物的配体取代 了溶度积较大沉淀物配体的反应: (A) 是; (B) 否。可以找答对的学生解释理由, 教师也可以进行讲 解, 从而帮助学生理解金属离子水解反应、溶解沉淀反应也可看作是配位反应的特例, 它们三者之 间有关联, 在一定条件下是可以相互转化的。大气光化学涉及的反应较多, 难以记忆, 可设置选择 
题将反应进行归类并指出异同点。如烷烃与自由基发生链反应的以下四个步骤中, 哪几个步骤也是 大多数烯烃发生的反应? (A) $\mathrm{RH}+\mathrm{HO} \cdot \rightarrow \mathrm{R} \cdot+\mathrm{H}_{2} \mathrm{O}$; (B) $\mathrm{R} \cdot+\mathrm{O}_{2} \rightarrow \mathrm{RO}_{2}$; (C) $\mathrm{RO}_{2} \cdot+\mathrm{NO} \rightarrow \mathrm{RO} \cdot+$ $\mathrm{NO}_{2}$; (D) $\mathrm{RO}+\mathrm{O}_{2} \rightarrow \mathrm{R}^{\prime} \mathrm{CHO}+\mathrm{HO}_{2}$ 。从而帮助学生理解烷烃、烯烃、环烃和芳香烃与自由基反应 的异同点, 把拥有相同规律的污染物特征及反应, 放在一起思考和记忆, 易构建成网状的知识体系, 从而能够根据所需及时提取, 促进前后学习的有效衔接。

随堂测验就是借助 “雨课堂” 设置选择题、填空题等, 让学生限时回答; 通过随机点名让学生 回答主观题, 从而可以检测学生对教学知识点的理解与掌握程度, 有助于教师进行针对性的指导。 在 “雨课堂” 中, 由于有答题的压力刺激, 学生为了避免 “答不上来” 的尴尬, 也会花更多注意力 在听课上; 不理解的地方学生可以按 “不懂” 按钮, 教师可对学生普遍不懂的问题进行反复讲解; 弹幕功能可以激发不爱说话的学生用手机发言, 活跃课堂气氛, 促进全班同学主动思考; 课堂红包 可以调动学生积极参与课堂, 提高学习兴趣;

课后, 教师还能通过平台向学生推送作业、视频、微课等资料, 拓展教学信息量, 可以帮助学 生进行总结归纳, 也可以推送环境化学前沿科研成果, 这对于扩展学生对课程知识认识的深度和广 度亦非常重要。引导学生了解相关理论知识在环境领域中的应用, 从而激发学生对科研和专业的热 爱。

“雨课堂”也可以帮助教师利用其大数据功能, 轻易获得出勤、课堂答题结果, 摆脱课堂上与 学生难互动、课后作业批改繁重的难题。“雨课堂” 可让学生手中的手机变成学习的教具, 使学习 从被动变为主动, 使学生从 “低头族” 变为 “抬头族” , 使教师从 “教书匠” 转变为 “教育设计 者” , 使教学资源从封闭到开放, 使教学从经验驱动的粗放管理到数据驱动的精益管理, 是一种非 常有效的教学模式。

\section{4 结语}

本文以环境化学与四大基础化学课程的教学衔接为研究内容, 重点解决了在环境化学教学过程 中由于与四大基础化学教学内容重复引起的教学效率降低、教学效果下降问题, 通过更新优化课程 内容, 实施多种教学方法, 采用雨课堂线上线下混合教学等多项举措有意识地带领学生寻找新、旧 知识之间的联系, 从学生认知结构中已有观念出发引导学生进行知识的建构, 提高学生对新知识的 吸收与掌握程度, 实现环境化学与四大基础化学的有效衔接。

\section{参 考 文 献}

[1] 汪群慧, 宋娜, 陈月芳, 常雁红, 吴川福. 大学化学, 2015, 30 (4), 11.

[2] 汪群慧, 王遂, 苏荣军. 环境化学. 第 2 版. 哈尔滨: 哈尔滨工业大学出版社, 2008 .

[3] 戴树桂. 环境化学. 第 2 版. 北京: 高等教育出版社, 2006.

[4] 大连理工大学无机化学教研室. 无机化学. 第 5 版. 北京: 高等教育出版社, 2006.

[5] 华东理工大学分析化学教研组, 四川大学工科化学基础课程教学基地. 分析化学. 第 6 版. 北京: 高等教育出版社, 2009.

[6] 汪小兰. 有机化学. 第 4 版. 北京: 高等教育出版社, 2005 .

[7] 傅献彩, 沈文霞, 姚天扬. 物理化学(上册). 第 5 版. 北京: 高等教育出版社, 2005.

[8] 于晓洋, 罗亚楠, 杨艳艳, 任红, 金华. 山东化工, 2017, 46 (22), 137. 